\title{
Variaciones del juego social en una camada de perros domésticos, con una madre con ansiedad por separación *
}

\author{
Alabarcez, M.N.; Koscinczuk, P.; Cainzos, R. \\ Cátedras de Bienestar Animal y Patología Médica, Facultad de Ciencias Veterinarias, UNNE, \\ Sargento Cabral 2139, Corrientes (3400), Argentina. Tel/Fax: 54-03783-425753. \\ E-mail:mnalabarcez@vet.unne.edu.ar.
}

\begin{abstract}
Resumen
Alabarcez, M.N.; Koscinczuk, P.; Cainzos, R.: Variaciones del juego social en una camada de perros domésticos con una madre con ansiedad por separación. Rev. vet. 21: 2, 93-98, 2010. El período de socialización del perro doméstico se extiende entre las tres y doce semanas de vida y proporciona al individuo capacidades para enfrentar el ambiente y establecer relaciones sociales, tanto intra como interespecíficas. En dicho lapso tiene lugar el juego que tendrá efectos beneficiosos o perjudiciales para el cachorro, dependiendo del contexto en el cual se desarrolla. En este trabajo se estudió el juego social de una camada $(n=6)$ de Weimaraner, comparando la respuesta de sus individuos frente a dos madres. Por un lado, la madre biológica, con patología comportamental de ansiedad por separación, y por el otro una perra Fox Terrier Smooth de perfil conductual normal. Ambas convivían con los cachorros en el mismo hogar. Se filmaron dos sesiones de juego con cada hembra interviniente en horario matutino. Se efectuó observación focal en cada individuo. Para comparar los efectos madre biológica o adoptiva, tanto para juego social como exploración ambiental, se registró sólo la frecuencia de las variables elegidas, sin considerar su intensidad ni duración. Para el análisis estadístico se utilizó el test no paramétrico de Kolmogorov-Smirnov a dos colas con un nivel de significación del 5\%. Los cachorros realizaron más actividades de contacto táctil, saltar, morder, aplastarse contra el suelo, sacudir objeto y olfatear en presencia de la perra adoptiva que ante la madre biológica $(p=0,01)$. No se encontraron diferencias significativas entre los sexos $(\mathrm{p}>0,05)$, sugiriendo que a esta edad machos y hembras responderían de manera similar. Si bien cabría la necesidad de ampliar el estudio sobre efectos de la crianza con madres adoptivas, este trabajo muestra que la intervención de perras normales favorece la coordinación de las actividades de juego y aprendizaje, generando estabilidad emocional y conductual en los cachorros.
\end{abstract}

Palabras clave: perro, socialización, comportamiento maternal, aprendizaje, bienestar animal.

\begin{abstract}
Alabarcez, M.N.; Koscinczuk, P.; Cainzos, R.: Social play variations in a domestic dog litter, with a biological mother with separation anxiety symptoms. Rev. vet. 21: 2, 93-98, 2010. The dog socialization period goes between the third and twelfth weeks of life and provides the individual the capacities to cope with the environment and establish the social inter and intra specifics bond relations. During this period games take place, and it is assumed that they may have either beneficial or detrimental effects, depending on the context. The social play development was evaluated in a Weimaraner 45 days litter $(n=6)$, to make a behavioral comparison between the biological mother with a separation anxiety pathology, and an alternative mother Fox Terrier Smooth with a normal behavioral pattern. Both bitches were living in the same place. Two play sessions with each female were filmed during the morning. Focal observations were performed on each individual to record social play and environmental exploratory activities. The variables were registered according to frequency of occurrence and comparing two main effects: biological vs alternative mother. For statistical analysis the nonparametric two-tailed Kolmogorov-Smirnov test was used, which showed a significance level of 5\%. The puppies performed several contact tactile activities: jump, bite, crush against the floor, and shake objects and sniff, more often with the alternative mother
\end{abstract}

Recibido: 10 setiembre 2010 / Aceptado: 15 octubre 2010

*Trabajo subsidiado por SGCYT-UNNE, presentado en el Congreso del Bicentenario: Bienestar Animal-Desafío para el próximo lustro, Buenos Aires, 2010. 
than with the biological one $(p=0.01)$. There were no significant differences between sexes for all the activities performed at this age $(\mathrm{p}>0.05)$. Despite the fact that further studies regarding the effect of nursing with alternative mothers are necessary, this trial show that a normal bitch helps to coordinate both play and learning activities to improve puppies development, promoting emotional and behavioral stability in response to environmental stressors.

Key words: dog, socialization, maternal behaviour, learning, animal welfare.

\section{INTRODUCCIÓN}

El perro doméstico es una especie que se caracteriza por su alto grado de socialización tanto intra como interespecífica. Al igual que en todas las especies de mamíferos sociales, el rol de la madre es fundamental para alcanzar un adecuado desarrollo cognitivo y conductual.

La conducta parental se desarrolla inmediatamente después del parto y está influenciada por diversas hormonas y neurotransmisores. El área preóptica media (estructura prosencefálica más importante para la conducta materna), el area tegmental ventral del mesencéfalo y la amígdala resultan estimuladas por el olor de las crías, la manipulación, los lamidos y los sonidos de llamadas ${ }^{6,17}$. El comportamiento de un individuo, al igual que otros eventos fenotípicos, depende de la genética y de la interacción ambiental. Cabe destacar que para el caso particular de los comportamientos sociales, el primer contexto ambiental está dado por la atención materna y los individuos que conforman el grupo. Esta relación materno-filial garantiza la perpetuidad de la especie.

Durante el período sensible del desarrollo conductual, la madre enseña a los cachorros los códigos sociales que rigen la conducta de la especie: impresión filial, socialización (intra e interespecífica), jerarquización e impronta sexual, entre otras $2,9,10,21,22$. Por otra parte, el grado de atención hacia una camada dependerá del temperamento, sensibilidad emocional y habilidades de la madre. Las patologías de comportamiento pueden afectar el desempeño materno, y de esta manera alterar el desarrollo conductual de los cachorros ${ }^{8,9}$.

Los perros presentan un repertorio conductual complejo y, al igual que en otras especies sociales con un elevado desarrollo cognitivo, el juego habría surgido especialmente como una herramienta de aprendizaje de las diferentes habilidades (exploración, caza, agresión, escape, desempeño sexual), con el objeto de aplicarlas posteriormente, fuera del contexto del juego, para situaciones de supervivencia ${ }^{1,2,4,12,15,29}$.

El objetivo de este trabajo fue comparar las respuestas de juego en una camada de Weimaraner de 45 días de edad, en presencia de dos hembras diferentes: la madre biológica, con una patología conductual -ansiedad por separación- y una madre adoptiva sin patologías conductuales que convivía en la misma casa.

\section{MATERIAL Y MÉTODOS}

Sujetos. El estudio se realizó con una camada de Weimaraner $(n=6)$ de 45 días de edad. Los cachorros fueron evaluados en una primera etapa junto a la madre biológica. Se trató de una hembra de 33 meses de edad, parto primerizo, que presentaba la patología conductual de ansiedad por separación (ante la ausencia de los propietarios: aumento de ansiedad, vocalización, hipersalivación, midriasis y respuesta motora excesiva). El comportamiento de juego con la madre biológica fue comparado con el de una hembra Fox Terrier Smooth de 20 meses, con patrón conductual normal que vivía en el mismo lugar.

Sitio de estudio. El estudio se realizó en un ambiente cerrado de $9 \mathrm{~m}^{2}$, con temperatura controlada, desde la 7:30 hasta las 13:30 horas. La duración de todas las sesiones fue de 6 minutos cada una, y fueron filmadas desde una habitación lateral a través de una ventana (semejante a una cámara Gesel), utilizando una cámara portátil Sony DCR-DVD 108. En el lugar de filmación sólo permaneció el experimentador con los sujetos de estudio; el propietario no se encontraba presente en el lugar.

Antes de comenzar las sesiones, se administró alimento balanceado y agua ad libitum, permitiendo que tanto los cachorros como la madre biológica exploraran libremente el lugar hasta mostrar signos de habituación al ambiente. Este procedimiento se repitió con la madre sustituta en la segunda etapa del registro de datos. Dentro de la habitación se distribuyeron tres objetos de juego: uno semejante a un pollo de plástico que al presionarlo hacía ruido, un hueso artificial de cartílago y una soga de $30 \mathrm{~cm}$ de longitud.

Obtención y registro de datos. Cada individuo fue identificado con un collar de diferente color para facilitar el muestreo focal ${ }^{20}$. El registro inicial de actividades se realizó teniendo en cuenta un etograma (Tabla 1) con variables que describieran el patrón de comportamiento y su grado de variación en, al menos, el diez por ciento de los individuos ${ }^{25,26}$. De esta manera, fueron seleccionadas tanto actividades dirigidas hacia las hembras intervinientes y a los hermanos (juego social), como la exploración del ambiente (actividades individuales). Además de las variables de comportamiento específicas para el análisis estadístico se consideraron 
Tabla 1. Etograma de las variables registradas durante las sesiones.

\begin{tabular}{lll}
\hline$*)$ & Comportamiento & Descripción \\
\hline 01 & Contacto Visual (CVIS) & Dos sujetos realizan contacto visual mutuo. \\
02 & Contacto Táctil (CTAC) & $\begin{array}{l}\text { Un individuo contacta con otro utilizando cualquier parte del cuerpo, lamiendo, chocando, } \\
\text { empujando o tocando a otro. }\end{array}$ \\
03 & Carrera (CARR) & $\begin{array}{l}\text { Corrida súbita desde un punto hacia otro, durante un episodio de juego. } \\
\text { Salto efectuado en el lugar o hacia otro sujeto, elevando las patas delanteras o las cuatro } \\
\text { simultáneamente. }\end{array}$ \\
04 & Saltar (SALT) & Un sujeto muerde a otro en cualquier parte del cuerpo al menos durante 3 segundos. Si el \\
& & Contacto duraba menos tiempo o solamente pellizcaban con los dientes, se lo consideró \\
& & CTAC. \\
06 & Tironear (TIRO) & $\begin{array}{l}\text { Dos sujetos tironean simultáneamente de un objeto con los dientes, con la intención de } \\
\text { ganarlo. } \\
\text { Apoyar el vientre y las cuatro patas en el suelo. }\end{array}$ \\
07 & Aplastarse (APLA) & $\begin{array}{l}\text { Un sujeto sacude rápidamente un objeto retenido entre los dientes, moviendo la cabeza de } \\
\text { un lado a otro }\end{array}$ \\
08 & Sacudir (SACU) & Morder repetidamente un objeto o plantas, simulando o efectuando masticación. \\
09 & Roer (ROER) & Recorrer el área con paso tranquilo, mirando hacia todas direcciones. \\
11 & Olfatear (OLFA) & $\begin{array}{l}\text { Oler detenidamente un punto específico del suelo, algún objeto del ambiente o del aire, } \\
\text { durante al menos } 3 \text { segundos. Si la acción duraba menos tiempo, se lo consideró parte de } \\
\text { EXPL. }\end{array}$ \\
\hline
\end{tabular}

Entre paréntesis se muestra la codificación utilizada para el análisis estadístico. Todas las variables se registraron en frecuencias de ocurrencia. $(*)$ Variables 01 a 06: consideradas para juego social. Variables 07 a 11: consideradas como actividades individuales.

otras actividades como alimentación, descanso y eliminación, aunque no correspondieran específicamente al etograma de juego, a los efectos de obtener un registro conductual completo de cada individuo.

Todas las variables se registraron en frecuencia de ocurrencia, no se tuvieron en cuenta la duración ni la intensidad. Se realizaron dos observaciones de cada sesión filmada, y luego se aplicó un índice de acuerdo entre observadores, dividiendo el valor mínimo sobre el máximo y multiplicando el resultado por 100. El acuerdo mínimo aceptado fue del 90 por ciento ${ }^{3}$. Por otro lado, se aplicó un índice de corrección sobre la frecuencia de las variables registradas para cada individuo. De esta manera, el índice de frecuencia para la variable 1, contacto visual (CVIS), sería:

$$
\text { índice CVIS }\left(x_{1}\right)=\left(\mathrm{n}_{\mathrm{x} 1}\right) / \Sigma\left(\mathrm{n}_{\mathrm{x} 1}+\mathrm{n}_{\mathrm{x} 2}+\ldots+\mathrm{n}_{\mathrm{x} 11}\right)
$$

Donde $\left(x_{1}\right)$ es el índice de frecuencia corregido para la variable $1 ; n$ es la frecuencia total registrada para la variable $x$; y los subíndices corresponden al número de cada variable, respectivamente. De esta manera, se obtuvo por cada individuo un índice de frecuencia de cada actividad (variable) con respecto al total de actividades que realizó a lo largo de toda la filmación.

Análisis estadístico. Los registros de ambas sesiones por individuo se utilizaron como réplicas, totalizando de esta manera 12 observaciones por cada madre. Se utilizó estadística no paramétrica para evaluar diferencias entre los grupos. Para los factores "madre interviniente" y "sexo" se aplicaron dos tests de Kolmo-
gorov-Smirnov. Todos los análisis se efectuaron con un nivel de significación de $\mathrm{p} \leq 0,05$.

\section{RESULTADOS Y DISCUSIÓN}

Variaciones según la perra interviniente durante el juego. Los cachorros mostraron una mayor frecuencia de actividades con la perra adoptiva (Figura 1) que con la madre biológica durante las sesiones de juego (test a dos colas Kolmogorov-Smirnov, $n_{\text {MAdoptiva }}=$ 12, $n_{\text {MBiológica }}=12$; CTAC: $K S=0,67$; SALT: $K S=0,83$; MORD: $K S=0,83$; APLA: $K S=0,67$; SACU: $K S=0,75$; OLFA: $K S=0,67$; para todas $p=0,01$ ).

La interacción con la madre biológica fue escasa. Los cachorros efectuaron señales visuales (CVIS) y

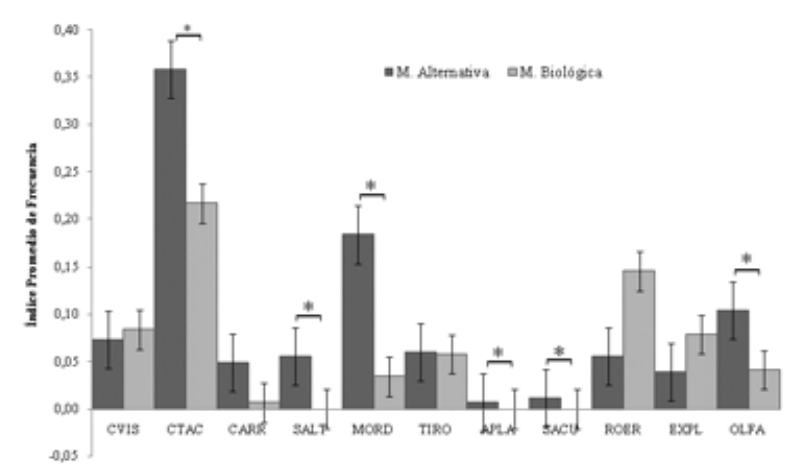

Figura 1. Índice promedio ( \pm error típico) para las frecuencias de actividades según la hembra interviniente. En asteriscos $p=0,01$. 
posturales con la cabeza y cuerpo, consideradas como una invitación al juego ${ }^{14}$. Al no obtener una respuesta por parte de la madre, algunos cachorros utilizaron un segundo tipo de demanda de atención; se colocaban delante de la madre, realizaban contacto táctil (CTAC), le mordían distintas partes del cuerpo (MORD) o intentaban mamar. Estos comportamientos -buscadores de atención-están específicamente dirigidos a un receptor que se encuentra desatento $u$ orientado en otra dirección sin la posibilidad de establecer contacto visual con el emisor ${ }^{14}$.

Mientras los cachorros intentaban establecer comunicación, la madre se hallaba vocalizando, hipersalivando, caminando en círculos, rascando la puerta y jalando el picaporte (Figura 2). Este conjunto de signos clínicos observados resultarían compatibles con el cuadro de ansiedad por separación, caracterizado entre otras cosas por midriasis, vocalización, hipersalivación, inapetencia y excesiva actividad motora ${ }^{16}$. Ante esta situación, los cachorros se agruparon en un rincón de la de la habitación, observando a la madre desde la distancia. Dos de los cachorros comenzaron a vocalizar y en todos ellos se observó midriasis, signos que podrían ser considerados como una respuesta rápida de estrés ${ }^{27}$.

En la segunda parte del estudio, se retiró a la madre biológica y los cachorros se colocaron con la madre adoptiva. Ésta fue la que tomó la iniciativa de contacto

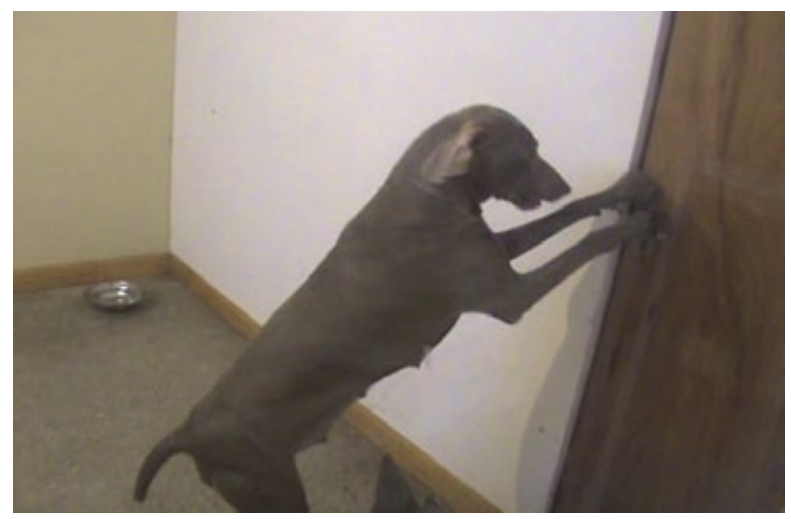

Figura 2. Detalle de la madre biológica durante la filmación, mostrando el cuadro de ansiedad por separación.

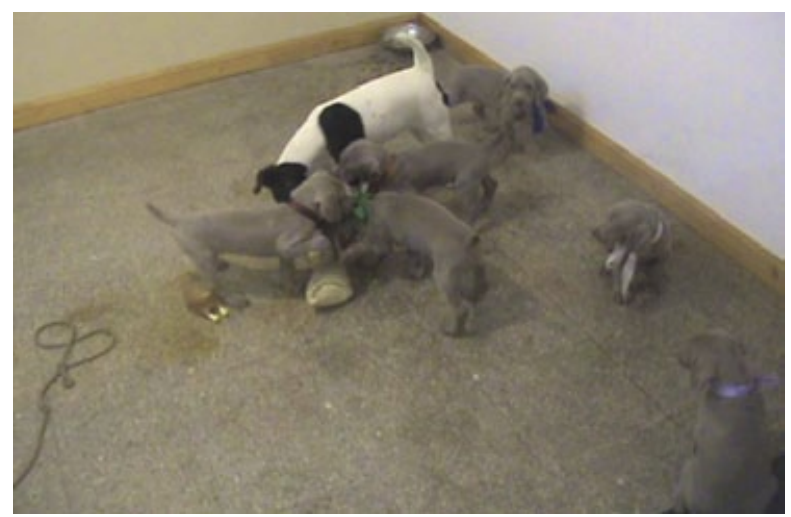

Figura 3. Detalle de la madre alternativa durante la filmación, compartiendo actividades con los cachorros.

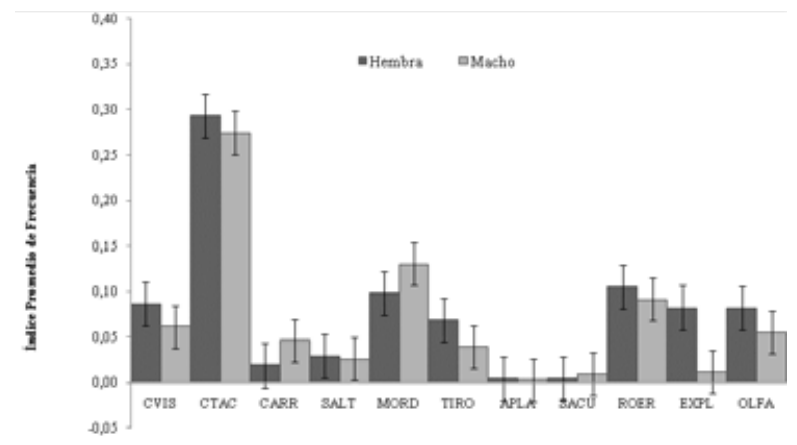

Figura 4. Índice promedio ( \pm error típico) para las frecuencias de actividades entre los sexos de los cachorros. Ninguna actividad mostró diferencias significativas $(p>0,05)$.

táctil, aproximándose hacia los cachorros, olfateándolos y lamiéndolos. Una vez que todos los cachorros se reincorporaron, como respuesta a la demanda de atención de la perra, ellos mismos comenzaron a realizar las señales de invitación al juego: contacto táctil (CTAC) y mordiscos (MORD). La perra permaneció en el centro del grupo (Figura 3), mientras los cachorros desplegaron secuencias de juego social entre hermanos, utilizando el contacto táctil, saltos (SALT), mordiscos y aplastándose (APLA) contra el suelo y sacudiendo los juguetes (SACU). Este incremento de actividades de la camada sugeriría mayores motivaciones de interacciones sociales con la madre adoptiva que con la biológica. Por otra parte, también realizaron una mayor exploración olfatoria (OLFA).

En el caso particular de nuestras observaciones, la patología conductual de la madre no permitió que los cachorros desarrollaran una secuencia normal de juego, lo que podría afectar a sus procesos de aprendizaje ${ }^{12}$. La precisión de eventos que se producen sobre el desarrollo fetal y neonatal resulta crítica para la integridad y función normal de los animales. Pequeños cambios en el esquema típico del desarrollo pueden ejercer efectos perdurables en el individuo ${ }^{19}$. La exposición a estresores en estos periodos afecta tanto la fisiología como el comportamiento de los animales $4,7,28$.

Durante el desarrollo se producen conexiones nerviosas (sinaptogénesis). A medida que el sistema nervioso recibe información del ambiente, se forman representaciones mentales de los estímulos. Estos procesos son rápidos y se forman en niveles altos de la organización cognitiva ${ }^{13}$. El hipocampo juega un rol importante para comparar los estímulos, el contexto, las asociaciones emocionales aprendidas y modula los efectos, participando en lo que se conoce como estabilidad emocional. La madre adoptiva utilizó el contacto táctil y presenció el juego de los cachorros coordinando sus actividades de manera efectiva.

En el caso de los cachorros de esta camada se observó una falta de cuidado por parte de la madre biológica. Esto podría generar diversas patologías conductuales como síndromes de hiperactividad-hipersensibilidad, estereotipias, diversos cuadros de ansiedad, miedo y 
posible agresión en estos individuos al alcanzar la adultez ${ }^{9,10,18}$. La privación materna puede afectar el modo en que un individuo percibe (de manera placentera o aversiva) el ambiente, alterando su respuesta conductual frente a nuevos estresores ${ }^{18}$.

Variaciones entre los sexos durante el juego. En este estudio no se observaron diferencias significativas para cualquiera de las actividades entre los sexos (test a dos colas Kolmogorov-Smirnov, $\mathrm{n}_{\text {Hembra }}=16, \mathrm{n}_{\text {Macho }}=$ 8; CVIS: $K S=0,19$, CTAC: $K S=0,25$; CARR: $K S=$ 0,38; SALT, MORD, TIRO, APLA, SACU y ROER: $K S$ $=0,31$; EXPL: $K S=0,44$; OLFA: $K S=0,38$; para todas $\mathrm{p}=0,20)$. Si bien hay autores que describen diferencias relacionadas con el sexo ${ }^{23,24,30}$, en este trabajo no fueron significativas, probablemente por el escaso número de individuos muestreados o a la edad de los individuos (45 días), por no haber alcanzado aún la maduración social y sexual (Figura 4).

Si bien cabría la necesidad de ampliar el estudio sobre los efectos de la crianza de madres adoptivas sobre cachorros de perros domésticos, este trabajo muestra de qué manera la presencia de otra perra puede coordinar las actividades de juego y aprendizaje de la camada, favoreciendo el período de formación de los cachorros. Este proceso resulta indispensable para generar una estabilidad emocional y conductual, mejorando las respuestas de los individuos hacia los estresores ambientales y por consiguiente optimizando su bienestar ${ }^{5,11}$.

Agradecimientos. Los autores agradecen a la familia Gaiad, propietarios de Delfina y Calandria, que formaron parte del trabajo. Asimismo, a la Ing. Agr. Belén Rossner por su asesoramiento estadístico.

\section{REFERENCIAS}

1. Bauer EB, Smuts BB. 2007. Cooperation and competition during dyadic play in domestic dogs, Canis familiaris. Anim Behav 73: 489-499.

2. Bekoff M. 2001. Social play behaviour. J Consc Studies 8: 81-90.

3. Bentosela M, Barrera G, Jakovcevic A, Elgier M, Mustaca AE. 2008. Effect of reinforcement, reinforcer omission and extinction on a communicative response in domestic dogs (Canis familiaris). Behav Proc 78: 464-469.

4. Boissy A, Manteuffel G, Bak Jensen M, Moe RO, Spruijt B, Keeling LJ, Winckler C, Forkman B, Dimitrov I, Langbein J, Bakken M, Veissier I, Aubert A. 2007. Assessment of positive emotions in animals to improve their welfare. Physiol \& Behav 92: 375-397.

5. Broom DM. 1999. Animal welfare: the concept and the issues. In: Attitudes to animals: views in animal welfare (Dolins FL Ed), Cambridge University Press, UK, p. 129142.

6. Carslon NR. 2002. Conducta reproductora. En: Fisiología de la conducta (Carslon NR Ed), Gayban Grafic, Barcelona, p. 378-420.
7. Casey R. 2002. Fear and stress. In: Manual of canine and feline behavioural medicine (Horwitz DF, Mills DS, Heath SH Ed), Ed. British Small Animal Veterinary Association (BSAVA), Gloucester (UK), p. 144-153.

8. Connolly PB. 2002. Reproductive behaviour problems. In: Manual of canine and feline behavioural medicine (Horwitz DF, Mills DS, Heath SH Ed), Ed. British Small Animal Veterinary Association (BSAVA), Gloucester (UK), p. 128-143.

9. Coll V. 2005. Evolución psíquica, educación y patologías del comportamiento. In: Neonatología y pediatría canina y felina (Prats A Ed), Inter-Médica, Buenos Aires, p. 451-475.

10. Dehasse J. 1994. Desarrollo sensorial, emocional y social del perro joven. Bull Vet Clin Ethol 2: 6-29.

11. Gazzano A, Mariti C, Notari L, Sighieri C, McBride EA. 2008. Effects of early gentling and early environment on emotional development of puppies. Appl Anim Behav Sci 110: 294-304.

12. Hall SL. 1998. Object play by adult animals. In: Animal play: evolutionary, comparative, and ecological perspectives (Bekoff M, Byers JA Ed), Cambridge University Press (UK), p. 45-60.

13. Halsberghe C. 2004. Developmental basis of emotional responses in companion animals. Proceed $10^{\text {th }}$ Europ Congr Companion Anim Behav Med (Cremona, Italia), p. 65-69.

14. Horowitz A. 2009. Attention to attention in domestic dog (Canis familiaris) dyadic play. Anim Cogn 12: 107-118.

15. Horváth Z, Dóka A, Miklósi Á. 2008. Affiliative and disciplinary behavior of human handlers during play with their dog affects cortisol concentrations in opposite directions. Horm and Behav 54: 107-114.

16. Horwitz DF. 2002. Separation-related problems in dogs. In: Manual of canine and feline behavioural medicine (Horwitz DF, Mills DS, Heath SH Ed), Ed. British Small Animal Veterinary Association (BSAVA), Gloucester (UK), p. 154-163.

17. Insel TR, Young LJ. 2001. The neurobiology of attachment. Nat Rev Neurosci 2: 129-136.

18. Latham NR, Mason GJ. 2008. Maternal deprivation and the development of stereotypic behaviour. Appl Anim Behav Sci 110: 84-108.

19. Lay DC. 2000. Consequences of stress during development. In: The biology of animal stress (Moberg GP, Mench JA Ed), CABI Publishing (Oxford, UK), p. 249-267.

20. Lehner PN. 1996. Handbook for ethological methods, $2^{\circ}$ ed, Cambridge University Press (UK), p. 672.

21. Manteca Vilanova X. 2003. Etología clínica veterinaria del perro y gato, $3^{\mathrm{a}}$ ed, Multimédica, Barcelona, p. 9-87.

22. Pal SK, Ghosh B, Roy S. 1998. Agonistic behaviour of free-ranging dogs (Canis familiaris) in relation to season, sex and age. Appl Anim Behav Sci 59: 331-348.

23. Pal SK. 2008. Maturation and development of social behaviour during early ontogeny in free-ranging dog puppies in West Bengal, India. Appl Anim Behav Sci 111: 95-107.

24. Pal SK. 2010. Play behaviour during early ontogeny in free-ranging dogs (Canis familiaris). Appl Anim Behav Sci 126: 140-153. 
25. Rooney NJ, Bradshaw JW. 2002. An experimental study of the effects of play upon the dog-human relationship. Appl Anim Behav Sci 75: 161-176.

26. Rooney NJ, Bradshaw JW. 2003. Links between play and dominance and attachment dimensions of dog-human relationships. J Appl Anim Welfare Sci 6: 67-94.

27. Ulrich-Lai YM, Herman JP. 2009. Neural regulation of endocrine and autonomic stress responses. Nat Rev Neurosci 10: 397-409.

28. Veissier I, Boissy A. 2006. Stress and welfare: two complementary concepts that are intrinsically related to the animal's point of view. Physiol \& Behav 42: 429-433.
29. Ward C, Bauer EB, Smuts BB. 2008. Partner preferences and asymmetries in social play among domestic dog, Canis lupus familiaris, littermates. Anim Behav 76: 11871199.

30. Wilsson E, Sundgren PE. 1998. Behaviour test for eightweek old puppies-heritabilities of tested behaviour traits and its correspondence to later behaviour. Appl Anim Behav Sci 58: 151-162.

\section{Revista Veterinaria obtuvo el máximo nivel de categorización del CAICYT-CONICET}

Tras el pertinente proceso de evaluación según criterios de calidad editorial, en setiembre de 2005 CAICYT-CONICET ha clasificado a nuestra publicación con Categoría 1 (nivel superior de excelencia), con lo cual pasa a integrar el Catálogo Latindex (folio 14022). La Dirección de Revista veterinaria agradece a quienes colaboraron para obtener tan importante distinción. Ver: http://www.latindex. unam.mx/busquedas/catalogotitulo.html 